(1)

CrossMark

\title{
The effect of mTOR inhibitors on respiratory infections in lymphangioleiomyomatosis
}

\author{
Andrew M. Courtwright, Hilary J. Goldberg, Elizabeth Petri Henske and \\ Souheil El-Chemaly
}

Affiliation: Division of Pulmonary and Critical Care Division, Brigham and Women's Hospital, Boston, MA, USA.

Correspondence: Souheil El-Chemaly, Thorn Biosciences Research Building, Room 805, Boston, MA 02115 E-mail: sel-chemalyapartners.org

@ERSpublications

Treating LAM patients with mTOR inhibitors does not increase respiratory infections and may be protective http://ow.ly/10EjIt

Cite this article as: Courtwright AM, Goldberg HJ, Henske EP, et al. The effect of mTOR inhibitors on respiratory infections in lymphangioleiomyomatosis. Eur Respir Rev 2017; 26: 160004 [https://doi.org/ 10.1183/16000617.0004-2016].

ABSTRACT Lymphangioleiomyomatosis (LAM) is a destructive cystic lung disease. Mammalian target of rapamycin (mTOR) inhibitors are the primary treatment for LAM but it is unknown whether these immunosuppressing medications increase the risk for or the severity of respiratory infections in LAM patients.

We searched multiple databases for original articles that reported the rate of respiratory infections in LAM patients treated with mTOR inhibitors or placebo. We calculated incidence rates for respiratory infections in these groups and incidence rate ratios for respiratory infections and severe respiratory infections in mTOR inhibitors treated versus placebo treated patients.

11 studies were included. There were 294 patients in the treatment groups and 93 patients in the placebo groups. Among subjects in placebo arms, the incidence rate of respiratory infections was 58.8 per 100 patient-years (95\% CI 35.3-82.3 per 100 patient-years). The incidence-rate ratio (IRR) for respiratory infection among treated subjects was 0.71 (95\% CI 0.50-1.02; $\mathrm{p}=0.06$ compared to placebo subjects). The IRR for severe respiratory infections among treated subjects was 1.56 (95\% CI 0.43-8.55; $\mathrm{p}=0.52$ ).

We found that respiratory infections are common in patients with LAM. Importantly, treatment with mTOR inhibitors does not increase the incidence of these infections and may be protective.

\section{Introduction}

Lymphangioleiomyomatosis (LAM) is a destructive, cystic lung disease caused by the proliferation of cells with mutations in the tuberous sclerosis complex (TSC) 1 or TSC2 genes [1]. Because of the ability of these cells to metastasise, LAM has been characterised as a low-grade neoplasm [2]. Respiratory failure is a principal cause of death in sporadic and TSC-associated LAM although lung function decline cannot easily be predicted based on phenotypic or biological parameters. LAM patients, however, appear to be at high risk for infections, which, in turn, may contribute to their clinical trajectory. For example, in the

Received: Jan 222016 | Accepted after revision: March 252016

Support statement: Support for this research was provided by a grant from the National Institutes of Health (5T32HL007633-30), the Department of Defense (TS130031), the Anne Levine LAM research fund, and the Lucy J. Engles TSC/LAM Research Program. Funding information for this article has been deposited with the Open Funder Registry.

Conflict of interest: Disclosures can be found alongside this article at err.ersjournals.com

Provenance: Submitted article, peer reviewed.

Copyright OERS 2017. ERR articles are open access and distributed under the terms of the Creative Commons Attribution Non-Commercial Licence 4.0. 
Multicenter International LAM Efficacy of Sirolimus (MILES) trial, a randomised double-blind placebo controlled clinical trial, the 43 subjects in the placebo group had 121 pulmonary or upper respiratory events over 1 year [3]. Other randomised trials have reported respiratory infections in one-third to one-half of placebo-arm LAM subjects $[4,5]$.

Mammalian target of rapamycin (mTOR) inhibitors were originally utilised as immune suppressant agents after solid organ transplantation. The use of mTOR inhibitors for treating LAM and other TSC-related complications, although at lower doses than in transplantation, has raised concerns that the immunosuppressive effects of these medications may increase the rate and/or severity of respiratory infections. This concern, in turn, may lead clinicians to have a lower threshold for antibiotic initiation and to continue antibiotics for a longer duration in their LAM patients receiving mTOR inhibitors. However, by slowing or reversing the underlying LAM-cell mediated pulmonary destruction, it is possible that mTOR inhibitors may actually stabilise lung architecture, leading to a decreased rate of pulmonary infections.

Individual studies reporting the rate of respiratory infections in LAM patients have been relatively small, with variable follow-up time, and have found a wide incidence of respiratory infections [3-5]. The objective of this meta-analysis was to review the current literature to assess the incidence rates of respiratory infections in LAM patients who are and are not undergoing treatment with MTOR inhibitors and to determine whether treatment is associated with an increased rate of respiratory infections and/or severe respiratory infections.

\section{Methods}

\section{Information sources}

We searched Medical Literature Analysis and Retrieval System Online, Journal Storage, Google Scholar, and Embase for English language studies between 2005 and 2015. We used the following key words in multiple combinations: lymphangioleiomyomatosis, LAM, tuberous sclerosis, adverse event, infection, respiratory infection, pneumonia, everolimus, mTOR, mammalian target of rapamycin and placebo. We reviewed the reference section of included articles to identify additional studies not found in our initial search. We performed the last literature search on January 1st 2016.

\section{Selection of publications}

One author (A.M. Courtwright) performed the initial eligibility assessment, although any concerns about eligibility were resolved by consensus between all authors. We initially screened studies using title and abstract and then reviewed full articles to assess for eligibility for inclusion. We excluded studies that focused on TSC patients alone. We included all randomised and non-randomised studies that reported adverse events in subjects undergoing treatment or in the placebo arm in the qualitative synthesis. For the final meta-analysis, however, we selected only studies that reported the number of patients who had one or more respiratory infections.

\section{Data collection process}

We developed a data extraction sheet, which we further refined after the initial search. We extracted the following data from each study: author name and year, study type, number of subjects, number of subjects with LAM, baseline mean percent predicted forced expiratory volume in $1 \mathrm{~s}\left(\mathrm{FEV}_{1}\right)$, number of subjects who withdrew, type of treatment (everolimus or sirolimus) or placebo, and length of follow-up. We also recorded the number of patients who experienced one or more respiratory infections, defined as upper respiratory infections, sinusitis, pneumonia, lower respiratory tract infections, bronchitis or pharyngitis. Finally, we recorded the number of patients who experienced a severe respiratory infection defined as grade 3 or higher or identified as severe (requiring hospitalisation, life-threatening or resulting in disability) by the study definitions.

Because of the mix of study designs we did not use a standardised quantitative instrument to rank study quality. Instead we used the Cochrane Collaborative's assessment tools for evaluating non-randomised and randomised studies $[6,7]$. These instruments assess risk of bias across multiple broad categories with the most significant risk of bias (low, high or unknown in randomised studies or low, moderate, serious, critical or no information in non-randomised studies) determining the overall risk of study bias. For each study, the focus of the risk of bias assessment was not on overall study design but on the risk of bias regarding diagnosis or reporting of respiratory infections. Finally, we cross-referenced author names and institutions to ensure that each data set was used in only one of the included studies.

\section{Statistical analysis}

The primary measure of effect was the incidence rate of respiratory tract infections in treatment and placebo groups. Because we did not have data on the exact time during the study period in which particular patients developed respiratory infections, we used a grouped data method to calculate person-years for each 
study. This method assumes that the rate of infection was constant during the study follow-up period. We combined individual statistics using a random effects model, assessing for heterogeneity using a $\mathrm{Q}$ statistic and an $\mathrm{I}^{2}$.

We then aggregated all study results to calculate the incidence-rate ratio (IRR) of having one or more respiratory infections with treatment versus placebo. We also aggregated all study results to calculate the IRR of having one or more severe respiratory infection with treatment versus placebo. We performed two post hoc IRR analysis, one excluding studies in which non-LAM TSC patients were enrolled and one excluding studies with the highest risk for bias. All analyses were conducted using Comprehensive Meta-Analysis (Version 3; BioStat Inc., Englewood, NJ, USA) and Stata (Version 14; Stata Corp, College Station, TX, USA).

\section{Results}

\section{Data extraction and literature review}

The initial search generated a total of 166 unique papers (figure 1). After reviewing titles and abstracts, we excluded 149 studies. We excluded a further six articles after reading full texts as these studies were primarily or exclusively focused on TSC patients and did not enrol sufficient LAM patients to meet our inclusion criteria. We included the remaining 11 studies in the qualitative synthesis, one of which reported the total number of respiratory infection events but not the number of patients with one or more respiratory infections [3]. The study authors, however, provided the primary data and all 11 studies were included in the final meta-analysis. Because the treatment arm of CHANG et al. [4] used doxycycline rather than an mTOR inhibitor, we included only the placebo group of this study in our analysis.

Three of the included studies were randomised placebo-controlled trials, five were non-randomised prospective open-label trials without a placebo group, two employed mixed prospective open-label and retrospective data analysis designs and one was a purely retrospective cohort study (table 1). The majority of studies were assessed to be low risk for bias in reporting respiratory infections [3-5, 8-12]. The two studies that had mixed prospective-retrospective design were considered serious risk as not all adverse events appeared to have been tracked prospectively $[13,14]$. Finally, the one purely retrospective study was considered critical risk because it excluded patients who had had an adverse event with sirolimus, including one with a respiratory event [15]. In addition, study data were collected from medical record review, raising the possibility that infectious events that occurred outside of routine clinical care were not captured.

There were a total of 387 patients in the included studies, 269 (69.5\%) of whom had LAM and the remainder of whom had non-LAM manifestations of TSC. Among all patients, there were 294 in the treatment groups, all of whom received either sirolimus $(186(63.3 \%))$ or everolimus $(108(36.7 \%))$. There were 93 in the placebo

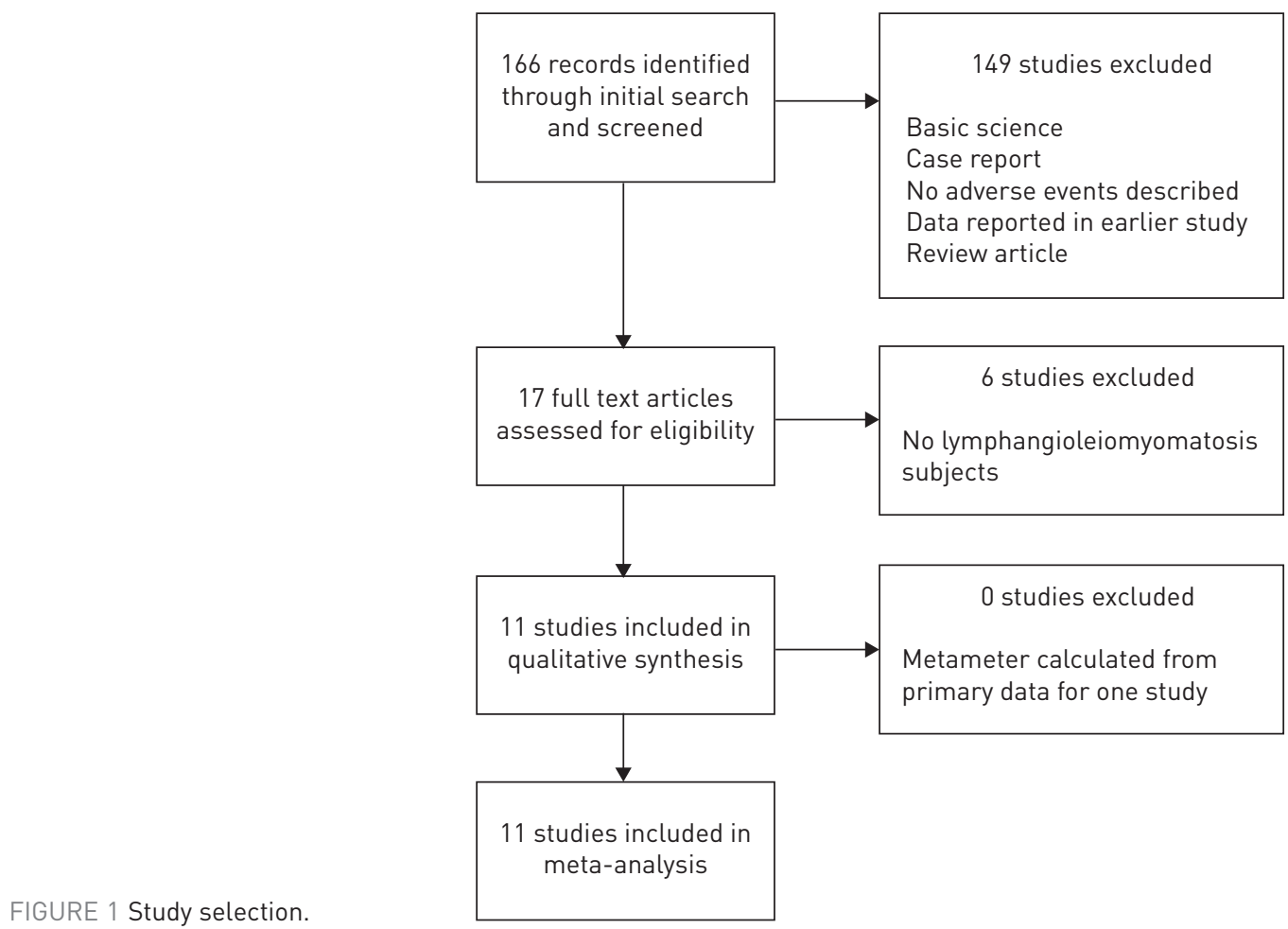




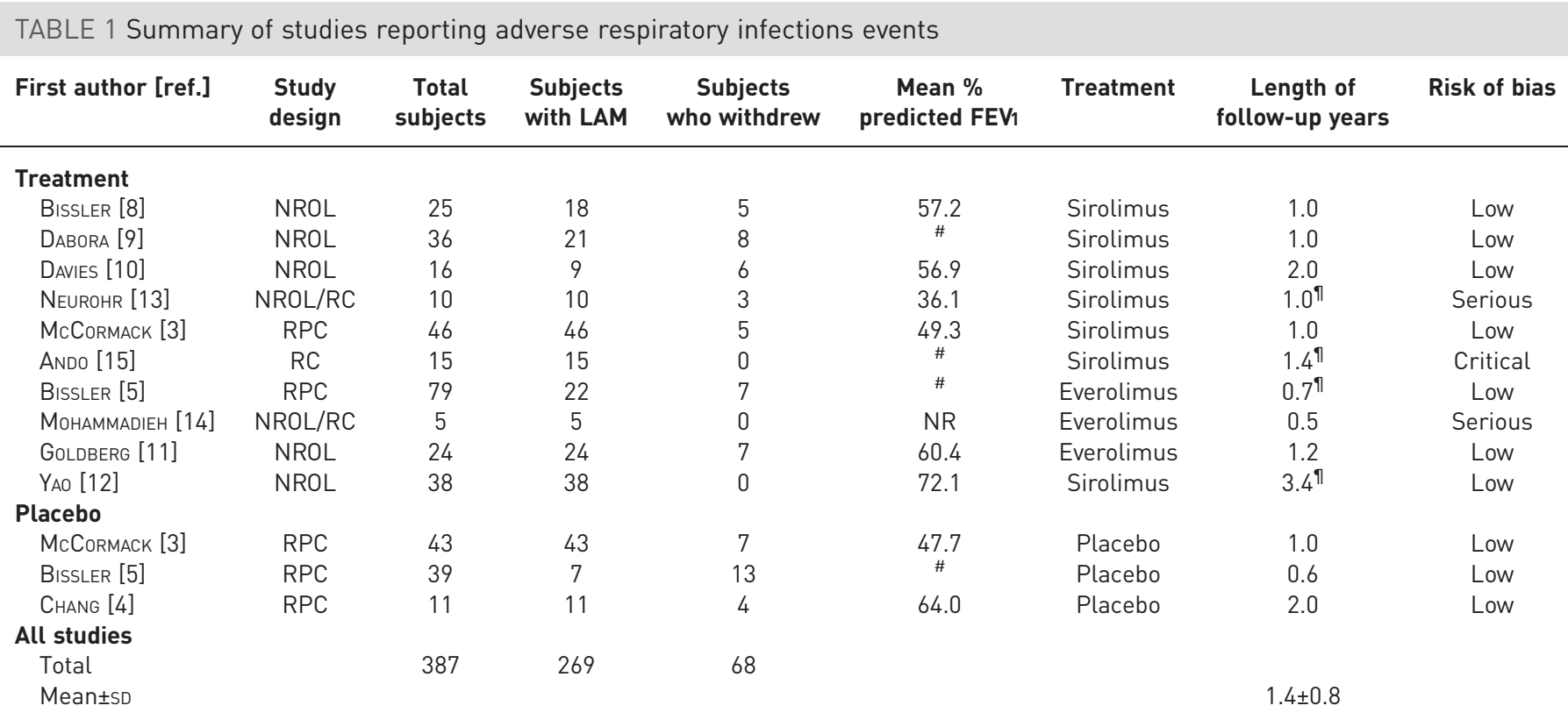

LAM: lymphangioleiomyomatosis; $\mathrm{FEV}_{1}$ : forced expiratory volume in $1 \mathrm{~s}$; NROL: non-randomised open label; RC: retrospective cohort;

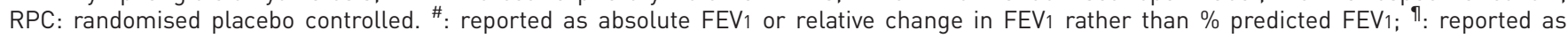
mean or median follow-up.

groups. Among studies reporting baseline percent predicted FEV1, the unweighted mean was 55.5 \pm 11.0 . Pulmonary exclusion criteria were variable, with some studies excluding patients requiring supplemental oxygen [10], with a post-bronchodilator $\mathrm{FEV}_{1}<30 \%$ predicted [11], or with large or recent chylous effusions $[3,4]$. There were $67(17.3 \%)$ total patients who withdrew during the study periods. Among patients receiving treatment, 41 (13.9\%) withdrew, most commonly because of adverse events (24 (58.5\%)). Among patients receiving placebo, 26 (28.0\%) withdrew, most commonly because of disease progression, adverse events, or to receive the study drug outside of the trial. The mean length of follow-up was $1.4 \pm 0.8$ years.

Most studies presented the number of patients with one or more upper respiratory infections, sinusitis, pneumonia, lower respiratory tract infections, bronchitis and/or pharyngitis according to standard adverse event definitions from the Medical Dictionary for Regulatory Activities or a similar tool. For one study, we calculated the number of patients during the first year treatment period who had one or more upper respiratory tract infections, lower respiratory tract infections, pneumonias, sinusitis or tonsillitis from primary data provided by the authors [3]. Two studies did not characterise the severity of respiratory infections and were excluded from the meta-analysis of infection severity $[12,14]$.

\section{Incidence rate}

Among subjects receiving mTOR inhibitors, the incidence rate of respiratory infections was 43.3 per 100 patient-years (95\% confidence interval (CI) 31.2-55.3 per 100 patient-years) (figure 2). There was significant and substantial heterogeneity among the studies $\left(\mathrm{I}^{2}=54.7, \mathrm{Q}=19.8, \mathrm{p}=0.02\right)$. Among subjects in placebo arms, the incidence rate of respiratory infections was 58.8 per 100 patient-years (95\% CI $35.3-82.3$ per 100 patient-years). There was not significant heterogeneity among studies $\left(\mathrm{I}^{2}=34.0, \mathrm{Q}=3.0, \mathrm{p}=0.22\right)$. Serious respiratory infections were rare in all study groups. Among subjects receiving mTOR inhibitors, the incidence rate of serious respiratory infections was 5.1 per 100 patient-years. Among subjects receiving placebo, the incident rate was 3.3 per 100 patient-years.

\section{Incidence rate-ratio}

The IRR for respiratory infection among treated subjects was not higher than placebo subjects (IRR $=0.71$ (95\% CI $0.50-1.02 ; \mathrm{p}=0.06)$. The IRR for severe respiratory infections among treated subjects was $1.56(95 \%$ CI $0.43-8.55 ; \mathrm{p}=0.52)$. Excluding BISSLER et al. [5], the only study in which the majority of patients had tuberous sclerosis rather than LAM, the IRR was 0.68 (95\% CI $0.45-1.07, p=0.08$ ) [5]. Excluding all four studies that enrolled non-LAM TSC subjects, the IRR was 0.69 (95\% CI 0.44-1.11, p=0.10). Excluding ANDO et al. 2013, the only study with a critical risk of bias, the IRR was 0.72 (95\% CI $0.51-1.05, \mathrm{p}=0.07)$ [15]. 


\begin{tabular}{|c|c|c|c|c|c|}
\hline \multirow[t]{2}{*}{ Study name } & \multirow{2}{*}{$\begin{array}{l}\text { Subgroup } \\
\text { within study }\end{array}$} & \multicolumn{4}{|c|}{ Statistics for each study } \\
\hline & & Rate & $\begin{array}{c}\text { Standard } \\
\text { error }\end{array}$ & $\begin{array}{c}\text { Lower } \\
\text { limit }\end{array}$ & $\begin{array}{c}\text { Upper } \\
\text { limit }\end{array}$ \\
\hline McCORMACK [3] & Placebo & 0.730 & 0.152 & 0.432 & 1.029 \\
\hline BISSLER [5] & Placebo & 0.673 & 0.180 & 0.321 & 1.026 \\
\hline \multirow[t]{2}{*}{ ChANG [4] } & Placebo & 0.375 & 0.153 & 0.075 & 0.675 \\
\hline & & 0.588 & 0.120 & 0.353 & 0.823 \\
\hline BISSLER [8] & Treatment & 0.778 & 0.208 & 0.370 & 1.185 \\
\hline DABORA [9] & Treatment & 0.286 & 0.095 & 0.099 & 0.472 \\
\hline DAVIES [10] & Treatment & 0.280 & 0.106 & 0.073 & 0.487 \\
\hline NEUROHR [13] & Treatment & 0.667 & 0.298 & 0.082 & 1.251 \\
\hline McCORMACK [3] & Treatment & 0.746 & 0.149 & 0.454 & 1.039 \\
\hline ANDo [15] & Treatment & 0.286 & 0.128 & 0.035 & 0.536 \\
\hline BISSLER [5] & Treatment & 0.589 & 0.113 & 0.367 & 0.811 \\
\hline MOHAMMADIEH [14] & Treatment & 1.000 & 0.707 & -0.386 & 2.386 \\
\hline GoLDBERG [11] & Treatment & 0.439 & 0.139 & 0.167 & 0.710 \\
\hline \multirow[t]{2}{*}{ YAO [12] } & Treatment & 0.288 & 0.058 & 0.175 & 0.401 \\
\hline & & 0.433 & 0.061 & 0.312 & 0.553 \\
\hline
\end{tabular}

Rate and $95 \% \mathrm{Cl}$

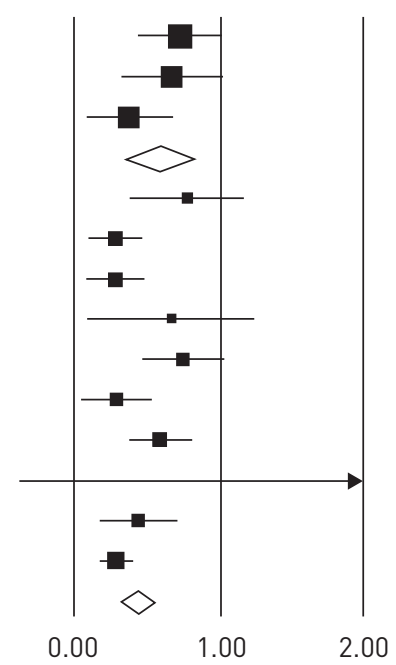

FIGURE 2 Incidence rates of respiratory infections in patients with lymphangioleiomyomatosis treated with placebo or mammalian target of rapamycin inhibitors.

\section{Discussion}

There has been concern among both patients and providers that the use of mTOR inhibitors such as everolimus or sirolimus in LAM patients may increase the frequency and severity of respiratory infections. Our primary findings in this meta-analysis were: 1) respiratory infections are common in patients with LAM (58.8 per 100 person-years); 2) the use of mTOR inhibitors does not appear to increase the incidence of respiratory infections and may be protective (IRR for all respiratory infections $0.71 ; \mathrm{p}=0.06$ ); 3) there is insufficient evidence to conclude whether the use of mTOR inhibitors is associated with increased incidence of severe respiratory infections.

What does a 58.8 per 100 patient-years incidence rate of respiratory infection mean clinically? If a clinician follows 100 LAM patients for a year and none of them are on mTOR inhibitors, approximately 59 will have a respiratory infection. In contrast, if the same clinician follows 100 LAM patients receiving mTOR inhibitors approximately 43 would be predicted to have a respiratory infection (a $27 \%$ reduction). Although this reduction in incidence approached but did not reach statistical significance $(\mathrm{p}=0.06)$, it is consistent with findings in MCCORMACK et al. [3], the largest, highest quality randomised placebo-controlled trial in this meta-analysis, that patients receiving sirolimus had reduced pulmonary or upper respiratory events. Despite the immunosuppressive effect of mTOR inhibitors, our study supports the concept that these medications may prevent respiratory infections. It is important to note that the relatively low incidence rate of severe respiratory infections in both treatment and placebo groups resulted in wide confidence intervals for the IRR of mTOR inhibitors on this outcome. We cannot exclude the possibility that mTOR inhibitors increase the rate of severe respiratory infections.

The mechanism through which mTOR inhibitors may prevent respiratory infection is unclear. It could be that delaying disease progression is, itself, preventative of infections. Alternatively, perturbations in the respiratory microbiome may play an under-appreciated role in LAM, as in other pulmonary diseases [1618]. In this setting, treatment with mTOR inhibitors may indirectly help restore or normalise the pulmonary microbiome [19]. Regardless, further research is needed to assess whether susceptibility to infection is related to severity of disease, an alternation of the pulmonary microbiome that is impacted by mTOR inhibitors, airway or lymphatic remodelling by inhibiting LAM cells, or an unexpected effect on the immune system at lower doses than used in transplantation.

The incidence risk of respiratory infection in LAM patients receiving placebo was higher than that reported in patients with interstitial lung disease enrolled in placebo groups in clinical trials. For example, using the same methodological assumptions as in this analysis, among patients with idiopathic pulmonary fibrosis (IPF) in the placebo group of ASCEND, a study of the anti-fibrotic pirfenidone, the incidence rate of respiratory infections was 22.5 per 100 patient-years [20]. Similarly, in the INPULSIS-1 and INPULSIS-2 trials of the tyrosine kinase inhibitor nintedanib, patients with IPF in the placebo group had an incidence rate of upper respiratory infections of 9.1 and 11.5 per 100 patient-years, respectively [21]. 
In this respect, LAM may be more similar to other obstructive lung disease, such as chronic obstructive pulmonary disease (COPD), in which the disease process is characterised by periods of relative stability punctuated by respiratory infection. For example, patients with COPD treated with placebo in studies of the phosphodiesterase inhibitor roflumilast had a 56.9 per 100 patient-years incidence rate of COPD exacerbations [22].

Although respiratory infections were common in all included studies, some LAM patients appeared more susceptible to frequent infections than others. For example, in the DAvies et al. [10] study, 5 patients were responsible for 16 upper respiratory infection events and many patients in the MCCORMACK et al. [3] study had multiple respiratory infections in both the placebo and treatment groups. Similarly there appear to be a group of patients that are relatively protected from infection. For example, YAO et al. [12] found that, over 3.4 years mean follow-up time, one-third of patients did not develop any upper respiratory tract infections. Interestingly, in the CHANG et al. [4] study, the incidence rate of respiratory infections in the treatment arm, which was doxycycline, was 71.4 per 100 patient-years. This suggests that doxycycline may not have the same protective effect as mTOR inhibitors. Other studies of doxycycline in LAM did not rigorously report respiratory events, making direct comparison difficult [23].

Our study has several limitations. First, although we were able to include all studies with untreated controls, the placebo group of our meta-analysis was drawn from only three studies, leading to relatively wide confidence intervals. Second, we calculated patient-years per study using the total number of subjects enrolled rather than the total number of subjects who completed the study. Many study withdrawals were related to adverse events and excluding these subjects may have led us to underestimate the number of patients with an infectious episode. Third, for studies that reported subtypes of respiratory infections such as upper respiratory infections, pneumonia, sinusitis, etc., we counted each as having occurred in a separate patient except where it was possible to determine which patients had multiple infections. It may have been the case, however, that the same patient was responsible for multiple events, which would have led to overestimating the number of patients who had one or more infections. Few studies, however, provided this detailed analysis and the majority of studies reported total number of patients with one or more respiratory infections. Fourth, we included four studies with a mix of both LAM and non-LAM TSC patients. Although LAM patients made up the majority of subjects in these studies, the inclusion of some non-LAM TSC subjects may have led us to over or underestimate the true incidence of respiratory infections. Excluding these studies from our IRR calculation did not significantly change our IRR estimate although it did widen the confidence intervals. Finally, we did not directly explore the reasons for the substantial heterogeneity in the incident rate of respiratory infections, particularly in the treatment groups. While this is unlikely related to reporting or capturing respiratory infections, particularly in the randomised and open-label trials, it may be due to differences in disease severity, the relative numbers of sporadic LAM and TSC-LAM subjects, or the use of sirolimus versus everolimus.

\section{Conclusions}

Respiratory infections are common in patients with LAM. Treatment with mTOR inhibitors does not appear to increase the incidence of these infections and may, surprisingly, be protective. Further research is necessary to determine whether mTOR inhibitors are associated with an increased risk of severe respiratory infections. Additional studies are warranted to determine the mechanism of the potential protective effect of mTOR inhibitors, which may involve the respiratory system, the immune system, the lymphatic system, epidemiologic factors and/or the pulmonary microbiome.

\section{Acknowledgements}

The authors would like to thank Francis McCormack (Dept of Pulmonary, Critical Care, and Sleep Medicine, University of Cincinnati, Cincinnati, OH, USA), for providing primary study data without which we would have had insufficient data for a robust placebo comparison group. We would also like to thank Bernard Rosner (Harvard Catalyst, Boston, MA, USA), for statistical analysis support.

\section{References}

1 Carsillo T, Astrinidis A, Henske EP. Mutations in the tuberous sclerosis complex gene TSC2 are a cause of sporadic pulmonary lymphangioleiomyomatosis. Proc Natl Acad Sci 2000; 97: 6085-6090.

2 McCormack FX, Travis WD, Colby TV, et al. Lymphangioleiomyomatosis: calling it what it is: a low-grade, destructive, metastasizing neoplasm. Am J Resp Crit Care Med 2012; 186: 1210-1212.

3 McCormack FX, Inoue Y, Moss J, et al. Efficacy and safety of sirolimus in lymphangioleiomyomatosis. $N$ Engl $J$ Med 2011; 364: 1595-1606.

4 Chang WY, Cane JL, Kumaran M, et al. A 2-year randomised placebo-controlled trial of doxycycline for lymphangioleiomyomatosis. Eur Respir J 2014; 43: 1114-1123.

5 Bissler JJ, Kingswood JC, Radzikowska E, et al. Everolimus for angiomyolipoma associated with tuberous sclerosis complex or sporadic lymphangioleiomyomatosis (EXIST-2): a multicentre, randomised, double-blind, placebocontrolled trial. Lancet 2013; 381: 817-824. 
6 Higgins JP, Altman DG, Gøtzsche PC, et al. The Cochrane Collaboration's tool for assessing risk of bias in randomised trials. $\mathrm{Br}$ Med J 2011; 343: d5928.

7 Sterne J, Higgins J, Reeves B. A Cochrane Risk of Bias Assessment Tool: for Non-Randomized Studies of Interventions (ACROBAT-NRSI). The Cochrane Collaboration. Available from https://sites.google.com/site/ riskofbiastool/. Date last accessed: January 14, 2016. Date last updated: September 23, 2014.

8 Bissler JJ, McCormack FX, Young LR, et al. Sirolimus for angiomyolipoma in tuberous sclerosis complex or lymphangioleiomyomatosis. N Eng J Med 2008; 358: 140-151.

9 Dabora SL, Franz DN, Ashwal S, et al. Multicenter phase 2 trial of sirolimus for tuberous sclerosis: kidney angiomyolipomas and other tumors regress and VEGF-D levels decrease. PLoS One 2011; 6: e23379.

10 Davies DM, de Vries PJ, Johnson SR, et al. Sirolimus therapy for angiomyolipoma in tuberous sclerosis and sporadic lymphangioleiomyomatosis: A phase 2 trial. Clin Cancer Res 2011; 17: 4071-4081.

11 Goldberg HJ, Harari S, Cottin V, et al. Everolimus for the treatment of lymphangioleiomyomatosis: a phase II study. Eur Resp J 2015; 46: 783-794.

12 Yao J, Taveira-DaSilva AM, Jones AM, et al. Sustained effects of sirolimus on lung function and cystic lung lesions in lymphangioleiomyomatosis. Am J Resp Crit Care Med 2014; 190: 1273-1282.

13 Neurohr C, Hoffmann AL, Huppmann P, et al. Is sirolimus a therapeutic option for patients with progressive pulmonary lymphangioleiomyomatosis? Respir Res 2011; 12: 66.

14 Mohammadieh AM, Bowler SD, Silverstone EJ, et al. Everolimus treatment of abdominal lymphangioleiomyoma in five women with sporadic lymphangioleiomyomatosis. Med J Aust 2013; 199: 121-123.

15 Ando K, Kurihara M, Kataoka $\mathrm{H}$, et al. The efficacy and safety of low-dose sirolimus for treatment of lymphangioleiomyomatosis. Resp Invest 2013; 51: 175-183.

16 Martin C, Burgel PR, Lepage P, et al. Host-microbe interactions in distal airways: relevance to chronic airway diseases. Eur Respir Rev 2015; 24: 78-91.

17 Molyneaux PL, Cox MJ, Willis-Owen SA, et al. The role of bacteria in the pathogenesis and progression of idiopathic pulmonary fibrosis. Am J Resp Crit Care Med 2014; 190: 906-913.

18 Simpson JL, Daly J, Baines KJ, et al. Airway dysbiosis: Haemophilus influenzae and Tropheryma in poorly controlled asthma. Eur Respir J 2016; 47: 792-800.

19 Pacheco-Rodriguez G, Kumaki F, Steagall WK, et al. Chemokine-enhanced chemotaxis of lymphangioleiomyomatosis cells with mutations in the tumor suppressor TSC2 gene. J Immunol 2009; 182: 1270-1277.

20 King Jr TE, Bradford WZ, Castro-Bernardini S, et al. A phase 3 trial of pirfenidone in patients with idiopathic pulmonary fibrosis. N Engl J Med 2014; 370: 2083-2092.

21 Richeldi L, du Bois RM, Raghu G, et al. Efficacy and safety of nintedanib in idiopathic pulmonary fibrosis. $N$ Engl J Med 2014; 370: 2071-2082.

22 Rabe KF, Bateman ED, O'Donnell D, et al. Roflumilast - an oral anti-inflammatory treatment for chronic obstructive pulmonary disease: a randomised controlled trial. Lancet 2005; 366: 563-571.

23 Pimenta SP, Baldi BG, Acencio MM, et al. Doxycycline use in patients with lymphangioleiomyomatosis: safety and efficacy in metalloproteinase blockade. J Bras Pneumol 2011; 37: 424-430. 